\title{
BULLETIN
}

DE

\section{l'Institut de Recherches Économiques et Sociales}

Périodique paraissant

hut fols par an

XII année

no 5 (1 er juillet 1946)

Fin novembre 1946

\section{S()MMAIRE}

LÉopold GÉNicot :

Etudes sur la construction des routes en Belgique . . . . . . . . . , . 495

('̇eORges VAN Hrike:

La réglementation de l'industrie de l'électricité aux Etats-Unis . . . . . . . 561

Roger DeHEM :

Un sophisme économique : le «salaire proportionnel » . . . . . . . . . . . . . .

INSTITUT DE RECHERCHES ECONOMIQUES ET SOGIALES

2 , RUE DES DOYENS

LOUVAIN 
Comite de Direction de l'Institut de Recherches Economiques et Sociales :

M. A.-E. Janssen, Professeur à la Faculté de Droit, Présiclent de l'Institut;

M. P. van ZEEI,AND, Professeur à la Faculté de Droit, Directeur de l'Institut;

M. F. Bardinuin, Professeur à la Faculté de Jroit, Secrétaire-Général de l'Institut;

M. J. LECIERCQ, Professeur à 'a Facalté cle Philosoplie et Lettres, Directeur des recherches sociales;

M. P. Rousseadx, Professeur à la Faculté de Droit, Directeur des recherches économiques;

M. F. (rRACCO, Professeur à l't,cole des Sciences Politiques et Sociales;

M. I.-H. Duprifz, Professeur à la Faculté de Droit;

M. Gे. Eyskens, Professeur à la Faculté de Droit;

M. P. DE BIE, Chargé de cours à la Faculté de Droit;

M. J.-E. MÉŕréns, Chargé de cours à la Faculté de Droit;

Le R. P. C. Van Gester, Maitre de conférences à l'Université.

\section{Rédaction du Bulletin :}

M. Y. Urbain, Professeur à l'École des Sciences Politiques et Sociales, Secretaire de 1 'Institut.

Les articles signés n'engagent que leur auteur.

\section{Abonnement annuel :}

\section{Belgique et Etranger : 200 Fr. belges.}

(Moyennant un supplément de $100 \mathrm{fr}$., les fascicules de Conjoncture sont expédiés au Congo belge par avion.)

\section{Vente au numéro :}

Fascicule 3 (Annuaire) : 120 Fr. belges.

Chacun des autres fascicules $(2,4,6,8$ : Conjoncture; $1,5:$ Economie; 7 : Sociologie) : 60 Fr. belges.

\section{Paiements :}

Bulletin de l'Institut de Recherches Economiques et Sociales, 2 , rue des Doyens, Louvain.

Compte Chèques no 5590.69 .

Comptes Banque : à Louvain, Banque de la Société Générale de Belgique; Kredietbank voor Handel en Nijverheid. 


\section{BULLE'TIN}

DE

\section{l'Institut de Recherches} Économiques et Sociales

\section{Pérlodique paraissant}

hult fols par an

\section{XII année}

no 8 (1er juillet 1946)

Fin novembre 1946

\section{SOMMAIRE}

LF́oPOLD GÉNICOT :

Etudes sur la construction des routes en

Belgique . . . . . . . . . , . 495

Georges vax Hegke :

La réglementation de l'industrie de l'élec-

tricité aux Etats-Unis . . . . . . . 561

Roger Dehem :

Un sophisme économique : le «salaire proportionnel») . . . . . . . . . 589

INSTITUT DE RECHERCHES ECONOMIQUES ET SOCIALES

2, RUE DES DOYENS

LOUVAIN 